${ }^{1}$ Department of Endocrine, General \& Vascular Surgery, Chair of Endocrinology, Medical University of Lodz, 62 Pabianicka Str, Lodz, Poland

2 Department of Molecular Bases of Medicine, I Chair of Internal Medicine, Medical University of Lodz, 251 Pomorska Str, Lodz, Poland

\title{
Evaluation of differential genes expression of ARHI, FAM129A, KCNQ1, CDH1, TIMP3, TFF3 and PTEN in thyroid tissue lesions and in biopsies obtained intraoperatively
}

\section{Background:}

Thyroid cancer is a serious epidemiological problem among endocrine diseases. Malignant transformation of nodular goiter (NG), can lead to the development of follicular adenoma (FA). The progression of $F A$ can lead to the transformation of benign neoplastic lesion into: papillary thyroid carcinoma (PTC), or more aggressive form - thyroid follicular cancer (FTC). The final differentiation of lesions (FA, PTC, FTC) is carried out post-operatively. Distinguishing follicular cell-derived thyroid tumors (FCDT) from nodular goiters on the molecular level by differential gene analysis could be helpfull epecially in case of underdetermined cytology (Bethesda III-IV) or FNAB with 'follicular neoplasm'. Therefore, it appears advisable to look for markers enabling the proper preoperative diagnosis.

\section{The aim of study: Comparison of expression levels of ARHI, FAM129A, KCNQ1, CDH1,} TIMP3, TFF3 and PTEN genes - as candidate biomarkers in differentiation of FCDT - in intraoperative FNAB and in mass of the lesion.

\section{Materials:}

56 specimens obtained postoperatively from patients who had undergone total thyroidectony with initial FNAB diagnosis: PTC or "follicular neoplasm". Patients aged 16 to 76 years (av. 49 years). After histopathological verification of the tissue samples:

PTC $(n=22)$, FTC $(n=5)$, FA $(n=6)$, NG $(n=23)$.

RNA isolated from: A) tissue lesion homogentes, B) FNAB of the indicated nodule, and C) macroscopically unchanged thyroid tissue - control.

$\begin{array}{cccl}\text { Histopathological classification } & \mathrm{n} & \% & \begin{array}{l}\text { Preoperative FNAB } \\ \text { cytological diagnosis }\end{array} \\ \text { nodular goiter (NG) } & 23 & 41 \% & \begin{array}{l}\text { PTC }-2, \\ \text { neoplasma folliculare }-21\end{array} \\ \text { follicular adenoma (FA) } & 6 & 10.5 \% & \text { neoplasma folliculare } \\ \text { carcinoma papillare (PTC) } & 22 & 39 \% & \begin{array}{l}\text { PTC } \\ \text { neoplasma folliculare - } 4\end{array} \\ \text { follicular carcinoma (FTC) } & 5 & 9.5 \% & \text { neoplasma folliculare }\end{array}$

Table 1. Histopathological classification of patients

BRAF mutations V600E were observed in 6 patients: 3 in PTC, 1 in FA, 2 in NG ( $p>0.05$, chi2 test).

$\mathrm{RQ}$ value for studied gene was calculated ( $\triangle \triangle \mathrm{CT}$ method) adjusted to GAPDH expression and to the calibrator expression level (macroscopically unchanged thyroid, $\mathrm{RQ}=1$ ).

FAM129A, KCNQ1, TFF3 and CDH1 genes were expressed on the comparable level in tumor tissue and material from biopsies - differences in RQ values between FNAB and tissue were not significant $(p>0.05$, Spearman's rank correlation coefficient)

Expression of:

\# KCNQ1 was elevated in NG, compared to cancer lesions.

\# FAM129A, CDH1 was the highest in PTC group

\# TFF3 was the highest in FTC group.

\section{Methods:}

\section{Total RNA isolation (Universal RNA Purification Kit - EURx)}

Qualitative and quantitative spectrophotometric analysis of RNA (RNA Nano LabChip, Agilent, BioPhotometer, Eppendorf).

Reverse transcription and BRAF V600E mutation analysis

$>$ mRNA expression level analysis (RQ) using Taq Man Low Density Arrays in 7900 HT Fast Real-Time PCR System (Applied Biosystems, USA).

Relative gene expression level assessment RQ $=\mathbf{2}^{-\Delta \Delta \mathbf{C T}}$

Statistical analysis (Statistica for Windows 10.0).

\section{Results:}

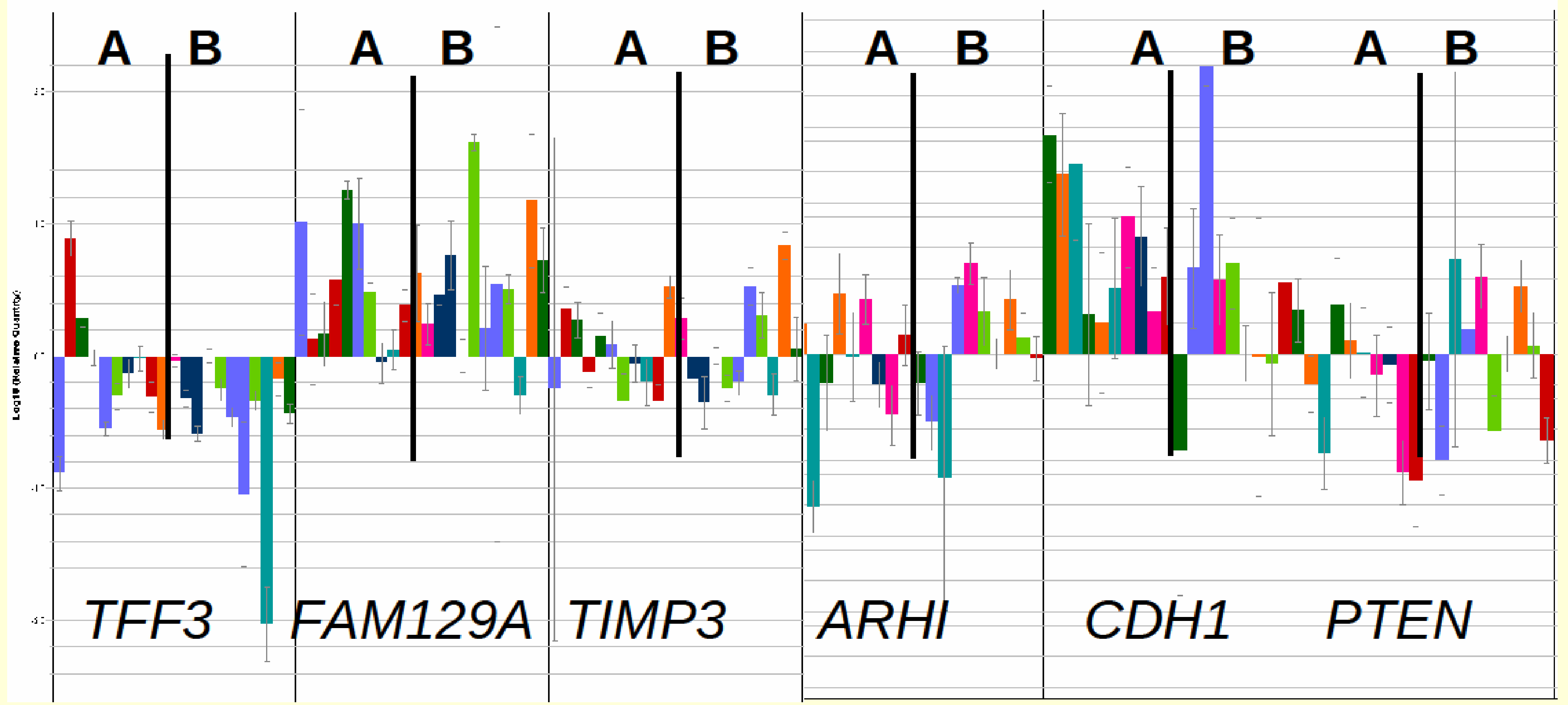

Figure 1. Example of differential expression analysis of TFF3, FAM129A, TIMP3, ARHI, CDH1, PTEN genes in PTC samples. A tissue homogenate, B- FNAB.

Differences in expression levels of TIMP3, ARHI and PTEN genes were observed between cancer tissue and FNAB material - $(p<0.05$, Spearman's rank correlation coefficient).

$A R H I$ and PTEN expression were significantly elevated in FNAB material when compared to tissue, TIMP3 decreased in biopsy.

Differences of gene expression of ARHI, TIMP3 and PTEN observed among histological subtypes, age groups and clinical features of the lesion exclude the possibility of using expression of these genes as markers for biopsies.

In this study we focused on search of genes of potential use as diagnostic markers in molecular analysis enhancing the traditional biopsies. Gene expression analysis of the following genes: TFF3, FAM129A, KCNQ1 and CDH1 in biopsy flushes may have diagnostic potential due to the expression on comparable levels in thyroid nodule biopsy and thyroid lesion after tyroidectomy. 